\section{Comment on: 'EULAR recommendations for the management of psoriatic arthritis with pharmacological therapies: 2019 update' by Gossec et al}

We read with great interest the published paper by Gossec et $a l^{1}$ which provided updated European League Against Rheumatism (EULAR) recommendations for pharmacological therapies for psoriatic arthritis (PsA). In this full-scale, evidence-based guideline, the authors proclaimed 6 overarching principles and 12 recommendations for PsA. Recently, the field of pharmacotherapy for PsA has progressed rapidly and is replete with competing clinical trials working on the efficacy of plenty of drugs. However, in light of some evidence on therapeutic efficacy still being scarce today, there are a few points in this study that are worth mentioning.

First, for PsA with relevant skin involvement, the authors recommended methotrexate (MTX) as the standard treatment superior to other conventional synthetic disease-modifying antirheumatic drugs (csDMARDs). However, the evidence on the efficacy of MTX remains limited to date. ${ }^{2}$ Furthermore, the medication trends for psoriasis in Asia seemed to be quite different from the EULAR recommendations. In our previous population-based study in Taiwan, we found that ciclosporin (CsA) was more commonly used for patients with psoriatic diseases in the past decade. ${ }^{3}$ Likewise, an epidemiological study from Korea reported higher use of CsA than MTX for patients with psoriasis, and the use of CsA showed a 32.2\% increase during the period of 2006-2015. The preference of CsA over MTX may be explained by the nature of CsA, which allows for easier monitoring and management of adverse effects. ${ }^{4}$ It is noteworthy that previous studies from Japan revealed improved quality of life for patients with psoriasis after lowdose and short-term use of CsA. ${ }^{56}$ Although CsA might pose unwanted side effects, nephrotoxicity caused by short-term use of CsA has been investigated to be reversible. Moreover, for patients with psoriasis, higher dose of CsA as induction therapy and lower dose of CsA as maintenance therapy have been reported to be a feasible way to control and diminish symptoms. The laboratory abnormalities found during induction therapy have been documented to return to normal ranges during maintenance therapy. ${ }^{7}$ Considering the insufficient evidence for MTX to be prior to other csDMARDs, we suggest that CsA should have a different weight on the discussion.

Second, the EULAR recommendations classified PsA into oligoarticular and polyarticular joint involvement. However, a previous study pointed out that the activity states of oligoarticular PsA cannot be accurately evaluated without assessing full 66/68 joint counts. The Disease Activity States of the PsA score, which is calculated by a comprehensive overview of the $66 / 68$ joint counts, has been proven to have high validity for clinical endpoints of PsA. ${ }^{8}$ As a result, to prevent misclassification and make treatment strategy for PsA more robust, thoroughly running through $66 / 68$ joint counts to evaluate active PsA instead of simply accounting for the affected joint counts is recommended.

Third, the authors provided the concept of cautious tapering of biologic disease-modifying antirheumatic drugs (bDMARDs) to the smallest effective dose when patients have reached sustained remission. Nonetheless, the article did not provide a valid algorithm for tapering down bDMARDs. A Denmark clinical trial published in 2019 displayed a tapering guidance for bDMARDs meanwhile maintaining stable disease activity for PsA. ${ }^{9}$ In our opinion, the EULAR recommendations should have an introduction on the tapering strategy in an effort to help alleviate the burden on patients with PsA.

Last but not least, the EULAR recommendations undoubtedly provided the most detailed and multifaceted guideline for the treatment of PsA. However, we think the therapeutic concerns for elderly patients with PsA deserve some attention. Based on a recently published study, there is obvious discrepancy in disease severity between late-onset and early-onset PsA. ${ }^{10}$ To date, evidence on pharmacological approaches to elderly patients with PsA is still lacking. Therefore, further research is needed to underpin therapeutic precautions and modification on the treatment dosage for elderly patients with PsA.

Chen-Yu Wei $\odot,^{1}$ Yung-Heng Lee, ${ }^{2,3,4,5}$ James Cheng Chung Wei $\odot^{6,7}$

${ }^{1}$ School of Medicine, Chung Shan Medical University, Taichung, Taiwan

${ }^{2}$ Department of Health Services Administration, China Medical University, Taichung, Taiwan

${ }^{3}$ Department of Public Health, China Medical University, Taichung, Taiwan ${ }^{4}$ Department of Orthopedics, Cishan Hospital, Ministry of Health and Welfare, Kaohsiung, Taiwan

${ }^{5}$ Department of Center for general education, National United University, Miaoli, Taiwan

${ }^{6}$ Graduate Institute of Integrated Medicine, China Medical University, Taichung, Taiwan

${ }^{7}$ Department of Medicine, Chung Shan Medical University Hospital; Institute of Medicine, Chung Shan Medical University, Taichung, Taiwan

Correspondence to Dr James Cheng Chung Wei, Department of Medicine, Chung Shan Medical University Hospital, Taichung 402, Taiwan; jccwei@gmail.com

Contributors C-YW and JCCW conceived and wrote the manuscript. Y-HL reviewed and commented on the manuscript. All authors approved the final version of the manuscript.

Funding The authors have not declared a specific grant for this research from any funding agency in the public, commercial or not-for-profit sectors.

Competing interests None declared.

Patient and public involvement Patients and/or the public were not involved in the design, or conduct, or reporting, or dissemination plans of this research.

Patient consent for publication Not required.

Provenance and peer review Not commissioned; internally peer reviewed.

(C) Author(s) (or their employer(s)) 2020. No commercial re-use. See rights and permissions. Published by BMJ.

\section{Check for updates}

To cite Wei C-Y, Lee Y-H, Wei JCC. Ann Rheum Dis Epub ahead of print: [please include Day Month Year]. doi:10.1136/annrheumdis-2020-218385

Received 23 June 2020

Accepted 29 June 2020

\section{S Linked}

- http://dx.doi.org/10.1136/annrheumdis-2020-218456

Ann Rheum Dis 2020;0:1-2. doi:10.1136/annrheumdis-2020-218385

\section{ORCID iDs}

Chen-Yu Wei http://orcid.org/0000-0002-1279-291X

James Cheng Chung Wei http://orcid.org/0000-0003-0310-2769

\section{REFERENCES}

1 Gossec L, Baraliakos X, Kerschbaumer A, et al. EULAR recommendations for the management of psoriatic arthritis with pharmacological therapies: 2019 update. Ann Rheum Dis 2020:79:700-12.

2 Gossec L, Coates LC, de Wit M, et al. Management of psoriatic arthritis in 2016: a comparison of EULAR and grappa recommendations. Nat Rev Rheumatol 2016;12:743-50 
3 Wei JC-C, Shi L-H, Huang J-Y, et al. Epidemiology and medication pattern change of psoriatic diseases in Taiwan from 2000 to 2013: a nationwide, population-based cohort study. J Rheumatol 2018;45:385-92.

4 Han JH, Lee JH, Han KD, et al. Epidemiology and medication trends in patients with psoriasis: a nationwide population-based cohort study from Korea. Acta Derm Venereol 2018;98:396-400

5 Okubo Y, Natsume S, Usui K, et al. Low-Dose, short-term ciclosporin (Neoral@) therapy is effective in improving patients' quality of life as assessed by Skindex-16 and GHQ-28 in mild to severe psoriasis patients. J Dermatol 2011:38:465-72

6 Hashimoto T, Kawakami T, Tsuruta D, et al. Low-Dose cyclosporin improves the health-related quality of life in Japanese psoriasis patients dissatisfied with topical corticosteroid monotherapy. Australas J Dermatol 2012;53:202-6.
7 Menter A, Gelfand JM, Connor C, et al. Joint American Academy of DermatologyNational psoriasis Foundation guidelines of care for the management of psoriasis with systemic nonbiologic therapies. J Am Acad Dermatol 2020:82:1445-86.

8 Coates LC, FitzGerald O, Gladman DD, et al. Reduced joint counts misclassify patients with oligoarticular psoriatic arthritis and miss significant numbers of patients with active disease. Arthritis Rheum 2013;65:1504-9.

9 Uhrenholt L, Schlemmer A, Hauge E-M, et al. Dosage reduction and discontinuation of biological disease-modifying antirheumatic drugs in patients with rheumatoid arthritis, psoriatic arthritis and axial spondyloarthritis: protocol for a pragmatic, randomised controlled trial (the biological dose optimisation (BIODOPT) trial). BMJ Open 2019;9:e028517.

10 Caso F, Tasso M, Chimenti MS, et al. Late-Onset and elderly psoriatic arthritis: clinical aspects and management. Drugs Aging 2019;36:909-25. 